\title{
- Characteristic polynomials of production matrices for geometric graphs
}

\author{
3 Clemens Huemer, Alexander Pilz†, Carlos Seara, Rodrigo I. Silveira*
}

\begin{abstract}
An $n \times n$ production matrix for a class of geometric graphs has the property that the numbers of these geometric graphs on up to $n$ vertices can be read off from the powers of the matrix. Recently, we obtained such production matrices for non-crossing geometric graphs on point sets in convex position [6]. In this note, we determine the characteristic polynomials of these matrices. Then, the Cayley-Hamilton theorem implies relations among the numbers of geometric graphs with different numbers of vertices. Further, relations between characteristic polynomials of production matrices for geometric graphs and Fibonacci numbers are revealed.
\end{abstract}

\section{Introduction}

A geometric graph on a point set $\mathcal{S}$ is a graph with vertex set $\mathcal{S}$ whose edges are straight-line segments with endpoints in $\mathcal{S}$. It is called non-crossing if no two edges intersect except at common endpoints. Here, we consider non-crossing geometric graphs on sets $\mathcal{S}$ of $n$ points in convex position for the following graph classes: triangulations, matchings, spanning trees, forests, spanning paths, and all geometric graphs on $n$ vertices. The numbers of these graphs are well known, see for instance the work of Flajolet and Noy [4]. Recently, in [6], we counted such geometric graphs by using an $n \times n$ matrix $A_{n}$, called production matrix, associated to the graph class. The numbers of these graphs on a certain number of vertices are then given by (a column of) powers of $A_{n}$. In order to derive a production matrix, first the graphs on $i \leq n$ vertices are partitioned according to the degree of a specified root vertex. Each part is counted in the elements of an $n$-element integer vector $\vec{v}^{i}$, and hence the sum of the elements gives the number of geometric graphs on $i$ vertices. The production matrix $A_{n}$ is such that $\vec{v}^{i+1}=A_{n} \vec{v}^{i}=A_{n}^{i+1-c} \vec{v}^{c}$, when starting with a vector $\vec{v}^{c}$ for a constant number of vertices, which will usually be $(1,0, \ldots, 0)^{\top}$. To find the matrix $A_{n}$,

*Departament de Matemàtiques, Universitat Politècnica de Catalunya, Barcelona, Spain. e-mail address: \{clemens.huemer, carlos.seara,rodrigo.silveira\}@upc.edu

${ }^{\dagger}$ Department of Computer Science, ETH Zürich, Switzerland alexander.pilz@inf.ethz.ch 


\begin{tabular}{|c|c|c|c|c|c|c|c|c|c|c|c|c|c|c|c|c|c|}
\hline $\bar{l} 1$ & 1 & 1 & 1 & 1 & $\overline{1}$ & $\overline{0}$ & 1 & 1 & 1 & 1 & 1 & $\overline{2}$ & 3 & 4 & 5 & 6 & 7 \\
\hline 1 & 1 & 1 & 1 & 1 & 1 & 1 & 0 & 1 & 1 & 1 & 1 & 1 & 2 & 3 & 4 & 5 & 6 \\
\hline 0 & 1 & 1 & 1 & 1 & 1 & 0 & 1 & 0 & 1 & 1 & 1 & 0 & 1 & 2 & 3 & 4 & 5 \\
\hline 0 & 0 & 1 & 1 & 1 & 1 & 0 & 0 & 1 & 0 & 1 & 1 & 0 & 0 & 1 & 2 & 3 & 4 \\
\hline 0 & 0 & 0 & 1 & 1 & 1 & 0 & 0 & 0 & 1 & 0 & 1 & 0 & 0 & 0 & 1 & 2 & 3 \\
\hline 0 & 0 & 0 & 0 & 1 & 1 & 0 & 0 & 0 & 0 & 1 & 0 & 0 & 0 & 0 & 0 & 1 & 2 \\
\hline \multicolumn{6}{|c|}{ (a) Triangulations } & \multicolumn{6}{|c|}{ (b) Matchings } & \multicolumn{6}{|c|}{ (c) Spanning trees } \\
\hline 1 & 1 & 1 & 1 & 1 & 1 & 1 & 1 & 1 & 1 & 1 & 1 & $(1$ & 1 & 1 & 0 & 0 & 0 \\
\hline 1 & 3 & 4 & 5 & 6 & 7 & 1 & 3 & 3 & 3 & 3 & 3 & 1 & 0 & 0 & 0 & 0 & 0 \\
\hline 0 & 1 & 3 & 4 & 5 & 6 & 0 & 2 & 4 & 4 & 4 & 4 & 0 & 1 & 0 & 0 & 0 & 0 \\
\hline 0 & 0 & 1 & 3 & 4 & 5 & 0 & 0 & 2 & 4 & 4 & 4 & 1 & 1 & 1 & 1 & 1 & 1 \\
\hline 0 & 0 & 0 & 1 & 3 & 4 & 0 & 0 & 0 & 2 & 4 & 4 & 0 & 1 & 1 & 0 & 1 & 1 \\
\hline 0 & 0 & 0 & 0 & 1 & 3 & 0 & 0 & 0 & 0 & 2 & $4)$ & 0 & 0 & 1 & 0 & 0 & $1)$ \\
\hline \multicolumn{6}{|c|}{ (d) Forests } & \multicolumn{6}{|c|}{ (e) Geometric graphs } & \multicolumn{6}{|c|}{ (f) Paths } \\
\hline
\end{tabular}

Figure 1: Production matrices for six different graph classes, for $n=6$. Matrix (f) is for paths on at most $n / 2$ points.

the graphs are implicitly arranged in a tree structure (called generating tree), s.t., for each graph on $i$ vertices and with root degree $j$, the number of its descendants on $i+1$ vertices with root degree $\ell$ (for each $\ell$ ) is known. Generating trees are the basis of the ECO method [1], and have been used to obtain matrix representations for combinatorial objects $[3,8]$. Here we omit how the production matrices for geometric graphs are obtained, and only refer to works by Hurtado and Noy [7] for a generating tree of triangulations, and by Hernando et al. [5] for a generating tree of spanning trees; and also to [6]. Figure 1 shows the obtained production matrices for the studied graph classes, for $n=6$. The matrix for triangulations was well-known, see e.g. [3, 8]. All matrices except for the matrix for paths are upper Hessenberg matrices. The different structure of the production matrix for paths, which is formed by four blocks, is due to a necessary distinction between paths with root vertex begin an endpoint of a path or an interior point. Also, for paths, the degree of the root vertex is defined in a different way, based on visibility (we omit the definition here). For all other graph classes, the degree is the number of edges incident to the root vertex.

The aim of the present work is to analyze these matrices by finding their characteristic polynomials. Previously, these polynomials were only known for triangulations [2], matchings, and spanning trees [6]. Here we determine the characteristic polynomial of the production matrices for geometric graphs, for forests, and for paths, solving the problem which was left open in [6]. An application of the characteristic polynomial of a production matrix follows from the CayleyHamilton theorem, which then implies a relation among the numbers of graphs with given root vertex degree. For example, via the production matrix for triangulations and $t_{n}(\lambda)$ one obtains the relation $\sum_{j=0}^{\lceil n / 2\rceil}(\underset{j}{n-j+1})(-1)^{n+j} C_{n-j}=0$ 
(a) triangulations

$t_{n}(\lambda)=\sum_{k=0}^{\lceil n / 2\rceil}\left(\begin{array}{c}n-k+1 \\ k\end{array}\right)(-1)^{n+k} \lambda^{n-k}$

(b) matchings

$m_{n}(\lambda)=\sum_{k=0}^{[n / 2\rceil} \sum_{j=0}^{n-k}\left(\begin{array}{c}n-k+1 \\ k\end{array}\right)\left(\begin{array}{c}n-k \\ j\end{array}\right)(-1)^{n+k} \lambda^{j}$

(c) spanning trees

$s_{n}(\lambda)=\sum_{k=0}^{n}\left(\begin{array}{c}2 k+2 \\ n-k\end{array}\right) \lambda^{k}(-1)^{k}$

(d) forests

$f_{n}(\lambda)=\sum_{k=0}^{n} \lambda^{k}(-1)^{k} \sum_{\ell=0}^{n} \sum_{j=0}^{\ell}\left(\begin{array}{c}\ell \\ j\end{array}\right)\left(\begin{array}{c}\ell \\ 2 \ell+j-n\end{array}\right)\left(\begin{array}{c}2 \ell+j-n \\ k+\ell+j-n\end{array}\right)$

(e) geometric graphs

$g_{n}(\lambda)=2^{n-1}+\sum_{k=1}^{n} \sum_{j=0}^{k-1}\left(\begin{array}{c}k-1 \\ j\end{array}\right)\left(\begin{array}{c}n-j \\ k\end{array}\right) 2^{n-k}(-1)^{k} \lambda^{k}$

(f) paths

$$
p_{n}(\lambda)=\sum_{k=0}^{n / 2-1}\left(\begin{array}{c}
n / 2-1 \\
k
\end{array}\right)(-1)^{n / 2+k+1} \lambda^{k}+\sum_{k=0}^{n / 2} 2 \frac{n-k}{n}\left(\begin{array}{c}
n / 2 \\
k
\end{array}\right)(-1)^{n / 2+k} \lambda^{n / 2+k}
$$

Figure 2: The characteristic polynomials of $n \times n$ production matrices for several graph classes. ${ }^{2}$

for the Catalan numbers $C_{i}$; we only refer to [6] for the full example.

Figure 2 shows the characteristic polynomials of the production matrices for the six studied graph classes. We give an outline of the proofs for geometric graphs and for forests in the following two sections.

\section{Geometric graphs}

Theorem 2.1 The characteristic polynomial $g_{n}(\lambda)$ of the $n \times n$ production matrix $G_{n}$ of geometric graphs is

$$
g_{n}(\lambda)=2^{n-1}+\sum_{k=1}^{n} \sum_{j=0}^{k-1}\left(\begin{array}{c}
k-1 \\
j
\end{array}\right)\left(\begin{array}{c}
n-j \\
k
\end{array}\right) 2^{n-k}(-1)^{k} \lambda^{k} .
$$

The proof is by induction on $n$, using the following two lemmas. First, we develop the determinant of $G_{n}-\lambda I_{n}$, where $I_{n}$ is the $n \times n$ identity matrix, to obtain a recurrence equation for $g_{n}(\lambda)$, and then to make induction work, we need to show a binomial identity.

Lemma 2.2 The characteristic polynomial $g_{n}(\lambda)$ of the matrix $G_{n}$ satisfies the recurrence relation

$$
g_{n}(\lambda)=(2-\lambda) g_{n-1}(\lambda)-2 \lambda g_{n-2}(\lambda) .
$$

Lemma 2.3 For $\ell \geq 2$ and $n \in \mathbb{N}$,

$$
\sum_{j=0}^{\ell-2}\left(\left(\begin{array}{c}
\ell-2 \\
j
\end{array}\right)\left(\begin{array}{c}
n-2-j \\
\ell-1
\end{array}\right)-\left(\begin{array}{c}
\ell-2 \\
j-1
\end{array}\right)\left(\begin{array}{c}
n-1-j \\
\ell-1
\end{array}\right)\right)=\left(\begin{array}{c}
n-\ell \\
\ell-1
\end{array}\right) .
$$

\footnotetext{
${ }^{2}$ The polynomials are also defined for $\lambda=0$ by setting the indeterminate form $0^{0}:=1$.
} 


\section{Forests}

Theorem 3.1 The characteristic polynomial $f_{n}(\lambda)$ of the $n \times n$ production matrix $F_{n}$ of forests is

$$
f_{n}(\lambda)=\sum_{k=0}^{n} \lambda^{k}(-1)^{k} \sum_{\ell=0}^{n} \sum_{j=0}^{\ell}\left(\begin{array}{l}
\ell \\
j
\end{array}\right)\left(\begin{array}{c}
\ell \\
2 \ell+j-n
\end{array}\right)\left(\begin{array}{c}
2 \ell+j-n \\
k+\ell+j-n
\end{array}\right) .
$$

Lemma 3.2 The characteristic polynomial $f_{n}(\lambda)$ of the matrix $F_{n}$ satisfies the recurrence relation

$$
f_{n}(\lambda)=(1-\lambda) f_{n-1}(\lambda)+(1-2 \lambda) f_{n-2}(\lambda)-\lambda f_{n-3}(\lambda) .
$$

To prove Theorem 3.1, we apply Riordan arrays as described for instance in [8]. Consider the infinite matrix

$$
M=\left(\begin{array}{ccccc}
1-\lambda & 1-2 \lambda & -\lambda & 0 & \ldots \\
1 & 0 & 0 & 0 & \ldots \\
0 & 1 & 0 & 0 & \ldots \\
0 & 0 & 1 & 0 & \ldots \\
\vdots & \vdots & \vdots & \vdots & \ddots
\end{array}\right)
$$

Let $\vec{w}^{0}$ be the vector $(1,0,0, \ldots)^{\top}$, and let $\vec{w}^{i}=\left(f_{i}(\lambda), f_{i-1}(\lambda), f_{i-2}(\lambda), \ldots, f_{1}(\lambda), 1,0,0, \ldots\right)^{\top}$. Then $\vec{w}^{i+1}=M \cdot \vec{w}^{i}$. Note that $\vec{w}^{n}$ is the first column of $M^{n}$. Using the notation from [8], we have that the $Z$-sequence is $\{1-\lambda, 1-2 \lambda,-\lambda, 0, \ldots\}$ and the $A$-sequence is $\{1,0, \ldots\}$; it follows that $h(t)=1$ and

$$
\begin{gathered}
d(t)=\frac{d_{0}}{1-t \cdot Z(t h(t))}=\frac{1}{1-t\left(1-\lambda+(1-2 \lambda) t-\lambda t^{2}\right)}= \\
=\sum_{k=0}^{\infty} t^{k}(t+1)^{k}(1-\lambda-\lambda t)^{k} ;
\end{gathered}
$$

the characteristic polynomial $f_{n}(\lambda)$ is then the coefficient of $t^{n}$ in this expression.

\subsection{Relation to Fibonacci numbers}

Denote with $F i b(n)$ the $n$-th Fibonacci number, where $F i b(1)=F i b(2)=1$, and $F i b(n)=F i b(n-1)+F i b(n-2)$ for $n>2$.

Corollary 3.3 The determinant of $F_{n}$ is the Fibonacci number Fib $(n+1)$.

Since the determinant of $F_{n}$ is $f_{n}(0)$, Corrollary 3.3 follows immediately from Equation (2) and $f_{0}(0)=f_{1}(0)=1$. Another family of matrices whose determinants are Fibonacci numbers can be found for instance in [9]. We remark that when we substitute $\lambda=0$ in the formula of Theorem 3.1 we also obtain, after simplification, the following well known identity. 
Corollary 3.4 The Fibonacci numbers satisfy the equation

$$
F i b(n+1)=\sum_{k=0}^{n}\left(\begin{array}{c}
k \\
n-k
\end{array}\right) .
$$

Also, the characteristic polynomial $f_{n}(\lambda)$ can be expressed recursively using Fibonacci numbers.

\section{Corollary 3.5}

$$
f_{n}(\lambda)+\lambda f_{n-1}(\lambda)=F i b(n+1)-\sum_{k=2}^{n} F i b(k+2) \lambda f_{n-k}(\lambda) .
$$

\section{Acknowledgments.}

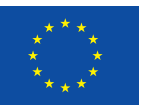

This project has received funding from the European Union's Horizon 2020 research and innovation programme under the Marie SkłodowskaCurie grant agreement No 734922 .

C. H., C. S., and R. I. S. were partially supported by projects MINECO MTM201563791-R and Gen. Cat. DGR2014SGR46. R. I. S. was also supported by MINECO through the Ramón y Cajal program.

\section{References}

[1] E. Barcucci, A. Del Lungo, E. Pergola, R. Pinzani. ECO: a methodology for the enumeration of combinatorial objects. J. Differ. Equations Appl. 5 (1999) 435490.

[2] G. Fairweather. On the eigenvalues and eigenvectors of a class of Hessenberg matrices. Siam Review 13(2) (1971) 220-221.

[3] E. Deutsch, L. Ferrari, S. Rinaldi. Production matrices. Advances in Applied Mathematics 34(1) (2005) 101-122.

[4] P. Flajolet, M. Noy. Analytic combinatorics of non-crossing configurations. Discrete Mathematics 204 (1999) 203-229.

[5] M. C. Hernando, F. Hurtado, A. Márquez, M. Mora, M. Noy. Geometric tree graphs of points in convex position. Discrete Applied Mathematics 93(1) (1999) 51-66.

[6] C. Huemer, C. Seara, R. I. Silveira, A. Pilz. Production matrices for geometric graphs. Electronic Notes in Discrete Mathematics 54 (2016) 301-306.

[7] F. Hurtado, M. Noy. Graph of triangulations of a convex polygon and tree of triangulations. Computational Geometry: Theory and Applications 13(3) (1999) 179-188.

[8] D. Merlini, M.C. Verri. Generating trees and proper Riordan Arrays. Discrete Mathematics 218 (2000) 167-183.

[9] G. Strang. Linear algebra and its applications. Brooks/Cole. Fourth Edition. 2006. 